\title{
Analysis of flame-flame interactions in premixed hydrocarbon and hydrogen flames
}

\author{
S. Trivedi, ${ }^{1, *}$ H. Kolla, ${ }^{2}$ J. H. Chen, ${ }^{2}$ and R. S. Cant ${ }^{1}$ \\ ${ }^{1}$ Department of Engineering, University of Cambridge, Cambridge CB2 1PZ, United Kingdom \\ ${ }^{2}$ Combustion Research Facility, Sandia National Laboratories, Livermore California 94550, USA
}

(Received 22 May 2020; accepted 25 September 2020;

published 17 November 2020)

\begin{abstract}
Flame-flame interactions are analyzed in twin hydrocarbon and hydrogen turbulent premixed flames. The interactions are identified with the help of Morse theory for critical points. Flame topology in the vicinity of critical points is analyzed and is categorized into four main groups, namely tunnel formation, tunnel closure, reactant pockets, and product pockets. The number of flame-flame interactions is presented in the form of histograms for the different flame cases. The relative frequency of occurrence of each type of topology changes with the turbulence intensity. In hydrocarbon flames, the fraction of product pockets and tunnel formation events increases with turbulence intensity, whereas the fraction of reactant pockets and tunnel closure events decreases. The results for hydrocarbon flames are compared with those for hydrogen flames and the differences are explained both qualitatively and quantitatively. An additional comparison is made between the number of flame-flame interactions observed for a single hydrocarbon flame against the number of interactions for twin hydrocarbon flames. It is found that having two flames in the domain does not significantly alter the number of interactions per flame, therefore indicating that the observed interactions are mainly self-interactions within individual flames.
\end{abstract}

DOI: 10.1103/PhysRevFluids.5.113201

\section{INTRODUCTION}

The increase in flame surface area induced by the action of turbulence [1-5] has been studied extensively using direct numerical simulation (DNS) [6-8]. Several closure models have been proposed for use within the Reynolds-averaged Navier-Stokes (RANS) or large eddy simulation (LES) approaches to account for these effects [9-14]. The local flame topology has been analyzed in various studies [15-18]. In high-intensity turbulence, the flame curvature increases to such an extent that self-interactions within the flame become inevitable [19-23]. The structures of the colliding flame surfaces merge and this may lead to significant changes in local burning rates [24,25].

In a study by Chen and Sohrab [24] the colliding flames were found to accelerate prior to merging. The chemistry in such flames was investigated by Echekki et al. [25]. In these studies $[24,25]$ interactions where the flames were propagating toward each other were called normal interactions. By contrast, counternormal interactions occur when the flames are propagating in opposite directions and are brought together by turbulent straining. Such interactions may involve local flame extinction and hence may result in the formation of pockets of partially burnt reactants $[21,26,27]$.

Flame-flame interactions can alter the flame surface area directly [19,28-31] and therefore alter the overall burning rate through the occurrence of regions of very high curvature and the burnout

*Corresponding author: st634@cam.ac.uk 
of pockets of reactants [19,20,32-38]. Flame-flame interactions are also responsible for inducing combustion-related noise [39-41]. Changes in flame area due to flame-flame interactions are still not rigorously accounted for in current models. Some attempts have been made to quantify the effects of flame-flame interactions on global flame properties [28,29,42]. However, the underlying physics of the effects of flame-flame interactions is still not fully understood. There is a need to understand the fundamentals of this phenomenon with a view to developing improved closure models.

Flame-flame interactions in narrow elongated flame channels may result in locations of very high flame curvature known as cusps [20]. The formation of cusps in turbulent flames was first suggested by Karlovitz et al. [1] and their dynamics were studied by Zel'dovich [32], who suggested cusps as the cure to unmitigated growth of instabilities within the flames. The dynamics of the formation of small isolated regions of reactants or products (known as pockets) in a flame have been analyzed mathematically in two dimensions (2D) by Kollmann and Chen [33] and in three dimensions by Trivedi et al. [38]. In a two-dimensional flame topology study by Chen et al. [19], the mechanism for pocket formation in a lean methane-air flame was investigated. It was found that pocket formation occurs in three stages: (1) collision of the reaction layers occurring at very short timescales, (2) cusp formation and recovery, and (3) burnout of the reactant pocket. It was also reported that strain and curvature play an important role in pocket formation.

A three-dimensional DNS study to find flame-flame interactions with the help of critical points for a pair of colliding hydrogen-air flames was carried out by Griffiths et al. [31]. This analysis was performed on the DNS data of Hawkes et al. [43] which consists of twin hydrogen flames propagating toward each other at Damköhler number $\mathrm{Da}=0.54(\mathrm{Da}+\mathrm{case})$ and $\mathrm{Da}=0.13(\mathrm{Da}-$ case). Another method to identify flame-flame interactions was developed by Dunstan et al. [21]. This method used wavelet transforms, and identified flame-flame interactions by comparing two flame surfaces taken from two different but closely spaced simulation time steps. The study [21] also reported changes in stretch rate due to flame-flame interactions. The present critical point method [31], on the other hand, can be applied on an instantaneous snapshot of the flame.

Experimental analysis of flame-flame interactions has been carried out by Tyagi et al. $[44,45]$ for single flame and dual flame configurations. Statistics were presented on the reactant side for normal interactions and on the product side for counternormal interactions for a range of operating conditions. Skiba et al. [46] has analyzed the flame surface density using experimental results in a round turbulent Bunsen flame, providing insight into the merging of flamelets and, hence, the destruction of flame surface density.

In the present paper, the numerical tools of Griffiths et al. [31] are adapted for the analysis of flame-flame interactions in a twin hydrocarbon flame setup at turbulence intensities of $u^{\prime}=10 s_{L}$, $20 s_{L}$, and $40 s_{L}$, where $s_{L}$ is the unstrained laminar flame speed. The interaction statistics of both hydrocarbon and hydrogen flame are analyzed for the entire range of reaction progress variable isosurfaces within the flames. It is possible to choose a single isosurface which may be taken to be representative of the flame surface as a whole. Indeed, the interaction statistics can be used to guide such a choice.

The main objective is to gain insight into the factors such as flame curvature, turbulence intensity, and turbulence dissipation rate which affect local flame surface interactions within hydrogen and hydrocarbon flames. The aim is to provide some insight and data to help to build and improve closure models that can account more rigorously for flame-flame interactions. At the same time, the analysis method is shown to be applicable to hydrocarbon as well as hydrogen flames. Analysis of the topology of the isosurface interactions can help to provide information about the influence on overall flame properties such as the consumption speed. Events such as mutual annihilation and pocket burnout take place rapidly and may have significant effects, for example, on the local flame area. Information on these local effects together with information on their frequency of occurrence can be used to estimate their global effects.

An additional comparison is drawn between the results for a single propagating hydrocarbon flame with those for a pair of hydrocarbon flames. Self-interactions in single hydrocarbon flames have been investigated previously by Trivedi et al. [23]. The presence of two flames in the domain 
results in approximately twice as many interactions as with a single flame, indicating that most observed flame-flame interactions in this study are, in fact, self interactions within individual flames. The next section of this paper describes the mathematical background including Morse theory for critical points. Section III describes the hydrogen and hydrocarbon datasets used in this study. The results obtained for these datasets are discussed in Sec. IV.

\section{TOPOLOGY OF FLAME-FLAME INTERACTION}

The number of flame-flame interactions can be quantified by using Morse theory of critical points [47] defined as the points on a surface at which the gradient is either zero or does not exist. A flame surface can be characterized with the help of a reaction progress variable $c$. A definition of $c$ using a suitable choice of species mass fraction is given as:

$$
c=\frac{Y_{\alpha}-Y_{\alpha R}}{Y_{\alpha P}-Y_{\alpha R}} .
$$

Here the subscript $\alpha$ denotes the chosen species, $R$ denotes reactants, and $P$ denotes products. The value of $c$ is required to rise monotonically from zero in the reactants to unity in the products. An isosurface of $c$ can be used as a marker for the flame surface.

When two flame surfaces interact, the gradient of the progress variable at the points of flameflame interaction vanishes. By definition, such points at which $\nabla c=0$ are critical points. The Taylor series expansion of the progress variable around a critical point located at $\underline{x}=\underline{a}$ is given by

$$
c(\underline{a}+\underline{x})=c(\underline{a})+\frac{1}{2} \underline{x}^{T} \cdot \underline{H}(c(\underline{a})) \cdot \underline{x}+\cdots,
$$

where $\underline{H}(c)$ is the Hessian matrix. The location of each critical point was found by first performing trilinear interpolation on each component of the gradient of the progress variable, based on its value at the eight neighboring solution points. If the interpolated value of the gradient passed through zero, then the corresponding location found using a linear approximation was used as the initial guess for a more refined solution using an algorithm based on Newton's method [48]. Since the DNS datasets were large, filtering methods were used to locate regions where the probability of finding critical points was high. More details about these steps can be found in Griffiths et al. [31]

The local topology in the vicinity of critical points can be described with the help of the eigenvalues of the Hessian matrix $\underline{H}(c)$. These eigenvalues $\left(\lambda_{1}, \lambda_{2}\right.$, and $\left.\lambda_{3}\right)$ can be used to evaluate the curvature along the three orthogonal principal axes defined by the corresponding eigenvectors $\left(e_{\lambda_{1}}, e_{\lambda_{2}}, e_{\lambda_{3}}\right)$. The eigenvalues define the local topology to second-order accuracy in $\underline{x}$. Shape factors $\theta$ and $\phi$ and mean curvature $\kappa$ can be derived using the eigenvectors (ordered as $\lambda_{1}>\lambda_{2}>\lambda_{3}$ ) according to

$$
\begin{gathered}
\theta=\frac{6}{\pi} \arctan \frac{\left(\lambda_{1}-2 \lambda_{2}+\lambda_{3}\right) / 6^{1 / 2}}{\left(\lambda_{1}-\lambda_{3}\right) / 2^{1 / 2}}, \\
\phi=\frac{2}{\pi} \arctan \frac{\left(\lambda_{1}+\lambda_{2}+\lambda_{3}\right) \cos (\theta \pi / 6) / 3^{1 / 2}}{\left(\lambda_{1}-\lambda_{3}\right) / 2^{1 / 2}} \\
\kappa=\left(\lambda_{1}^{2}+\lambda_{2}^{2}+\lambda_{3}^{2}\right)^{1 / 2}
\end{gathered}
$$

The shape factors fully define the local topology of the flame surface close to a critical point. Using the shape factors, Griffiths et al. [31] defined all possible topologies of flame-flame interactions and categorized them into four main groups, namely "reactant pocket" (RP), "tunnel closure" (TC), "tunnel formation" (TF), and "product pocket" (PP). The full map of all possible topologies is displayed in Fig. 1.

In Fig. 1, the blue color represents a lower value of $c$ and the red color represents a higher value of $c$. The flame always propagates toward the lower value of $c$. Reactant pockets are inward propagating topologically spherical pockets with reactants at a lower $c$ value surrounded by burnt products at a higher $c$ value and are therefore represented by the solid red color in the 


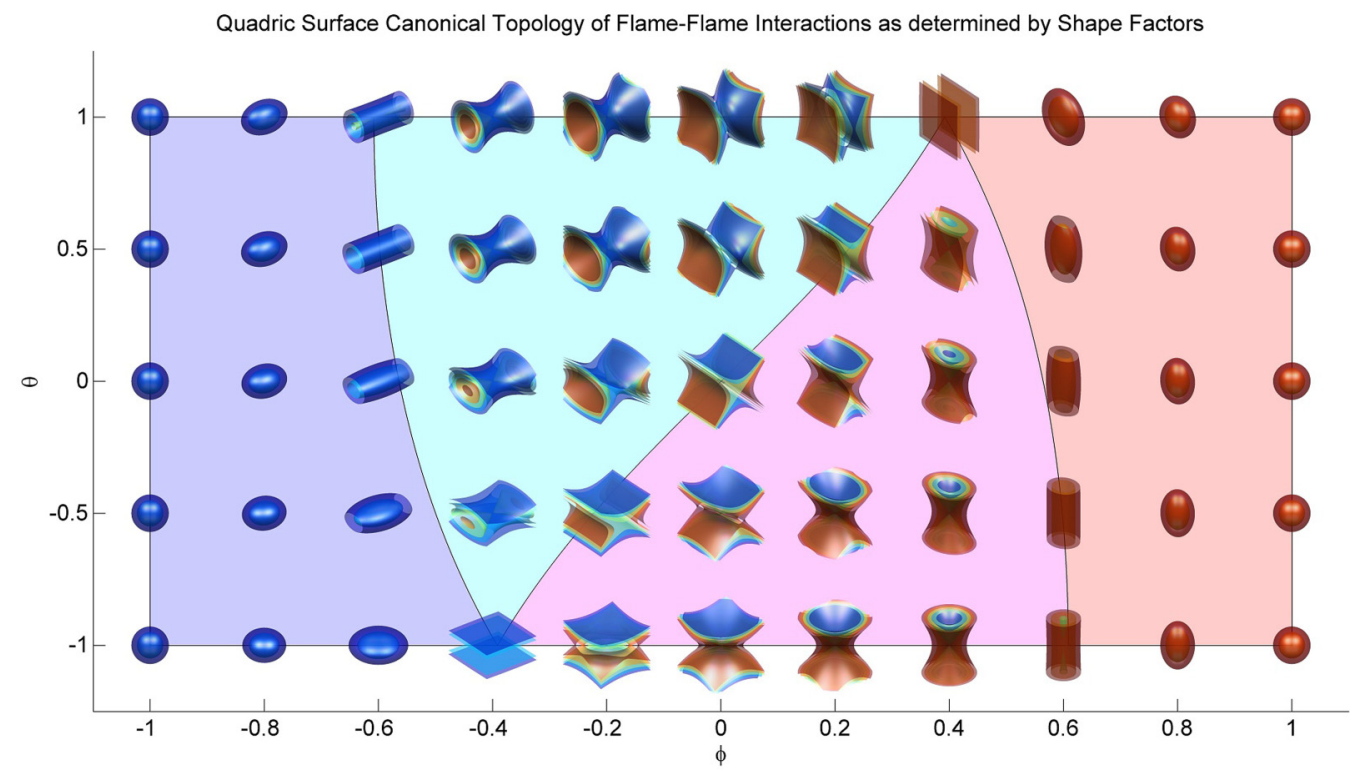

FIG. 1. The set of all possible flame-flame interaction topologies as determined by the shape factors [31].

figure. Likewise, product pockets propagate outward with burnt products at a higher $c$ value in the middle surrounded by unburnt reactants at a lower $c$ value. Similarly, tunnel closure involves inward propagation of a topologically cylindrical flame, while tunnel formation involves outward propagation.

\section{DNS DATASETS FOR THE CURRENT STUDY}

Two DNS datasets are used in the present work. First, a hydrocarbon flame dataset was created using the DNS code Senga2 [49]. The governing equations for the reacting flow are solved numerically in their 3D compressible form with 10th-order spatial and 4th-order temporal discretisation schemes. Boundary conditions are imposed using the Navier-Stokes characteristic boundary condition (NSCBC) formulation. Combustion chemistry may be treated using either complex or simplified chemical reaction mechanisms. The current hydrocarbon-air dataset is generated using a single-step reaction mechanism in which the chemical reaction formula is simply $R \rightarrow P$, where $R$ is the reactant and $P$ is the product. For this mechanism, the progress variable is simply defined as $c=Y_{P}$. This scheme preserves the transport and thermal properties of hydrocarbon flames. It is tuned to replicate the propagation speed of a stoichiometric methane-air flame, i.e., $39 \mathrm{~cm} / \mathrm{s}$, and also to capture the corresponding thickness of the reaction zone.

A field of homogeneous isotropic turbulence of desired intensity computed a priori is mapped on to the domain and is then allowed to evolve as part of the solution. Three different cases were simulated with the turbulence intensity of the initial mixture varied from $u^{\prime}=10 s_{L}$, to $u^{\prime}=20 s_{L}$ and, finally, $u^{\prime}=40 s_{L}$, where $s_{L}$ is the unstrained laminar burning velocity for the methane-air flame. The integral length scale $\ell_{0}$ was kept the same for all cases at $\ell_{0}=2 \delta_{L}$, where $\delta_{L}$ is the thermal thickness of an unstrained laminar flame, given by

$$
\delta_{L}=\frac{T_{P}-T_{R}}{(|d T / d x|)_{\max }}
$$

Here subscripts $R$ and $P$ denote temperature in unburnt reactants and burnt products. 


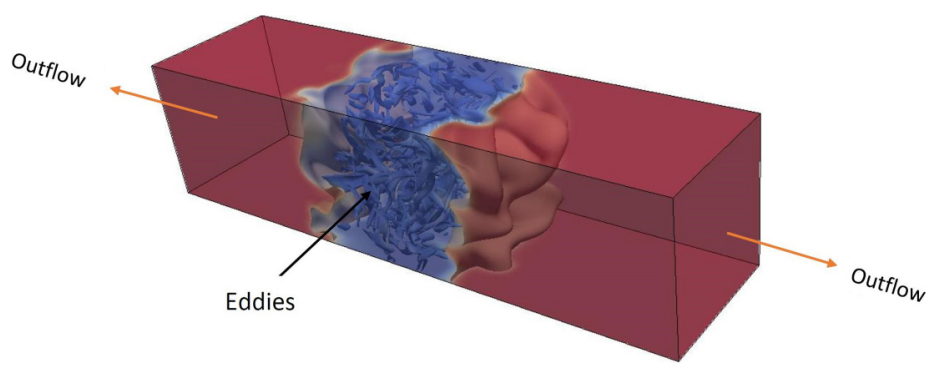

FIG. 2. Schematic diagram of the twin hydrocarbon flame configuration. Outflow boundary conditions are set on the end faces of the domain, with periodic boundary conditions on all other faces. Blue contours in the middle represent the turbulent eddies.

Two premixed planar laminar flames are initialized facing each other, with their corresponding normal vectors (defined to point into the reactants) along the $x$ and $-x$ directions. They are set at distance $\sim 15 \delta_{L}$ apart. This allows for a sufficient number of large eddies of size $\ell_{0}=2 \delta_{L}$ to exist between them. The initial temperature and pressure were set as $T_{0}=300 \mathrm{~K}$ and $p_{0}=1 \mathrm{bar}$ respectively. The Lewis number is kept equal to unity. A schematic of the twin flame configuration is shown in Fig. 2. The flames become wrinkled by the action of turbulence as they propagate toward each other. Eventually, the flames collide and mutually annihilate each other.

Both the $x$ direction boundaries make use of NSCBC reacting outflow conditions to allow for the exit of the expanded burnt products. The boundaries are set as periodic in the $y$ and $z$ directions. There is no inflow of fuel in any direction and therefore the flames are only able to propagate into the fresh reactants in the middle of the domain. The initial flame profile and turbulence evolve together as the flames propagate toward each other. The planar flames become wrinkled under the influence of turbulence as seen in Fig. 2.

Apart from the turbulence intensity $u^{\prime}$, all other thermochemical and physical parameters are kept constant across the dataset. The Karlovitz number

$$
\mathrm{Ka}=\frac{u^{\prime}}{\lambda} \frac{\delta_{L}}{s_{L}} \sim\left(\frac{u^{\prime}}{s_{L}}\right)^{\frac{3}{2}}\left(\frac{\delta_{L}}{\ell_{0}}\right)^{\frac{1}{2}},
$$

based on the Taylor length scale $\lambda$ varies across the dataset as $u^{\prime}$ increases. Likewise, the Damköhler number, given as

$$
\mathrm{D} a=\frac{s_{L} \ell_{0}}{u^{\prime} \delta_{L}},
$$

decreases as $u^{\prime}$ increases. Some of the important parameters of this dataset along with the dimensionless numbers $\mathrm{Ka}$, Da, and $\mathrm{Re}\left(=\mathrm{Ka}^{2} \mathrm{Da}^{2}\right)$ are summarized in Table I.

TABLE I. Main parameters of the twin hydrocarbon flame configuration.

\begin{tabular}{llll}
\hline \hline & \multicolumn{2}{c}{$u^{\prime} / s_{L}$} \\
\cline { 2 - 4 } Parameters & \multicolumn{1}{c}{10} & 20 & 40 \\
\hline$\ell_{0} / \delta_{L}$ & 2.0 & 2.0 & 2.0 \\
$\eta(\mu \mathrm{m})$ & 26 & 18 & 13 \\
$\mathrm{Ka}$ & 22.36 & 63.25 & 178.9 \\
$\mathrm{Da}$ & 0.20 & 0.10 & 0.05 \\
$\mathrm{Re}$ & 20 & 40 & 80 \\
$x$ length $(\mathrm{mm})$ & 30 & 40 & 80 \\
\hline \hline
\end{tabular}



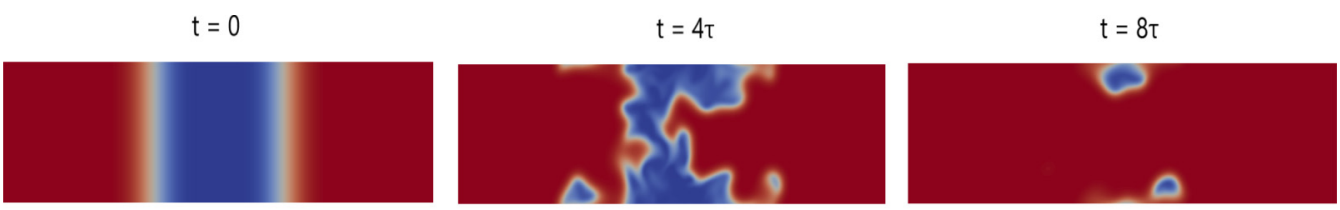

(a) $u^{\prime} / s_{L}=10$


(b) $u^{\prime} / s_{L}=20$


(c) $u^{\prime} / s_{L}=40$

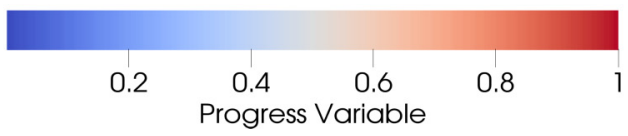

FIG. 3. Slices through the 3D domain of twin hydrocarbon flames at $t=0,4 \tau, 8 \tau$ in the left, middle, and right panels, respectively, for $u^{\prime} / s_{L}=10,20$, and 40 cases (top to bottom). The flames are seen to become wrinkled and eventually collide with each other.

The length of the domain in the $x$ direction varies for each case to keep the flames sufficiently distant from the boundaries. The lengths in the $y$ and $z$ directions remain constant at $5 \mathrm{~mm}\left(\sim 12 \delta_{L}\right)$. The domain was discretized using a uniform mesh with spacing $\Delta x=26 \mu \mathrm{m}$ and a fixed timestep $\Delta t=5 \mathrm{~ns}$ was used in all the simulations. Each simulation was run until the flames had fully interacted, which took a different amount of time for each case. The case with $u^{\prime}=10 s_{L}$ fully interacted in $t \simeq 12 \tau$, where $\tau$ is the eddy turnover time, defined as $\tau=\ell_{0} / u^{\prime}$. The higher-intensity case at $u^{\prime}=20 s_{L}$ took $t \simeq 20 \tau$ and the highest-intensity case at $u^{\prime}=40 s_{L}$ took $t \simeq 30 \tau$ to interact fully.

Figure 3 shows the results taken using this dataset with the turbulence intensity increasing from $u^{\prime}=10 s_{L}$ in the top row to $20 s_{L}$ in the middle row and $40 s_{L}$ in the bottom row. The snapshots are taken after $t=0,5 \tau$, and $10 \tau$, respectively. The colors represent the reaction progress variable based on product species mass fraction with values ranging from from zero (blue) to unity (red). The flames are clearly seen to be wrinkled by the effect of the turbulent flow.

The hydrogen-air dataset in this analysis is due to Hawkes et al. [43] consisting of two initially planar flames interacting with a turbulent rectangular slot jet at an equivalence ratio of 0.7 and with reactants preheated to $700 \mathrm{~K}$. A nine-species 21 -step reaction mechanism was used [50]. The 


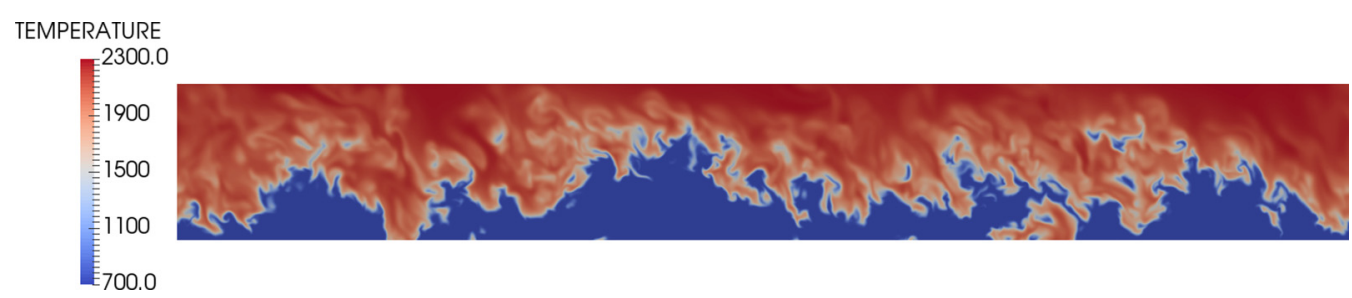

FIG. 4. Temperature field from a subset of the Da+ dataset from Hawkes et al. [43].

DNS code S3D [51] with eighth-order spatial finite-difference scheme and fourth-order RungeKutta time-stepping was employed to produce this dataset.

Two different Damköhler number cases called the $\mathrm{Da}-$ case $(\mathrm{Da}=0.13)$ and the $\mathrm{Da}+$ case $(\mathrm{Da}$ $=0.54)$ were analyzed. The domain contained $2400 \times 1600 \times 1800$ mesh points with physical size $16 H \times 20 H \times 12 H$, where $H$ is the width of the slot-jet. The value of $H$ was 5.4 and $2.7 \mathrm{~mm}$ and the mesh spacing was 36 and $18 \mu \mathrm{m}$ for the $\mathrm{Da}+$ and $\mathrm{Da}-$ cases, respectively. The integral length scale was $H / 3$ resulting in the value of $1.8 \mathrm{~mm}\left(\sim 3.6 \delta_{L}\right)$ for the $\mathrm{Da}+$ case and $0.9 \mathrm{~mm}\left(\sim 1.8 \delta_{L}\right)$ for the $\mathrm{Da}-$ case. Boundary conditions were set to be periodic in the streamwise and spanwise directions, with outflow in the direction perpendicular to the flames. It should be noted that the turbulence in this dataset is affected by mean shear, which can induce turbulent mixing within the flame structure [52]. A small subsection of the entire dataset was enough to find sufficient numbers of critical points for analysis. The temperature field for the $\mathrm{Da}+$ case of this subset is shown in Fig. 4. More details on this dataset can be found in Ref. [43].

All of the flames in the hydrocarbon and hydrogen datasets are in the thin reaction zone regime where the smaller scales of turbulence penetrate into the preheat zone but the reactants and products are separated by a continuous flame surface. No broken reaction zones were observed.

Contour slices of various isosurfaces of progress variable ranging from the leading to the trailing edge of the flame are shown in Fig. 5 for subsets of the Da+ (left) and $\mathrm{Da}-$ (right) cases. Here the progress variable was defined according to Eq. (1) using the mass fraction of $\mathrm{H}_{2}$. It is clear from the spacing between the blue contours (denoting lower values of progress variable) and the red contours (denoting higher values) that the preheat zone is disrupted by the action of turbulence. This feature of the flames has an important implication in the flame-flame interaction study. The isosurfaces are no longer parallel to each other $[20,35]$ and each of them is wrinkled somewhat independently. Thus, the observed flame-flame interactions are not always at the global flame level but occur locally within an isosurface of the progress variable.

\section{RESULTS AND DISCUSSION}

The statistics of flame-flame interactions obtained from the twin hydrocarbon flame dataset are presented first. The computational tools of Griffiths et al. [31] were applied to these flames to determine the number of critical points and, hence, the number of flame-flame interactions within the computational domain. The signs of the normalized eigenvalues of these critical points were used to identify the flame-flame interaction topology (see Sec. II).

Multiple snapshots were used starting at $t=2 \tau$ onward until the flames fully interacted in order to obtain a sufficient number of flame-flame interactions for all the hydrocarbon flame cases. The total number of interactions is presented in Fig. 6 in the form of histograms plotted against the progress variable $c$, showing the frequency of occurrence of critical points.

Different types of flame-flame interaction topologies are represented by different colors (see legend in Fig. 6). The histograms in the left column show the occurrence of critical points throughout the thickness of the flame, that is, $0.01<c<0.99$, whereas those in the right column are restricted to $0.3<c<0.99$ to reveal the detail in this range of progress variable. Note that there is a sharp rise in the number of observed critical points as the turbulence intensity is increased, i.e., moving 


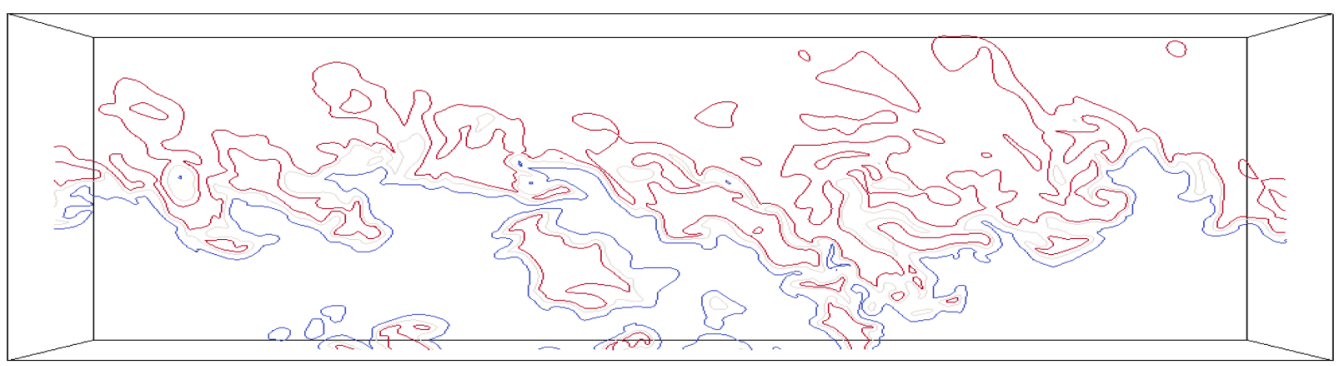

(a) $\mathrm{Da}+$

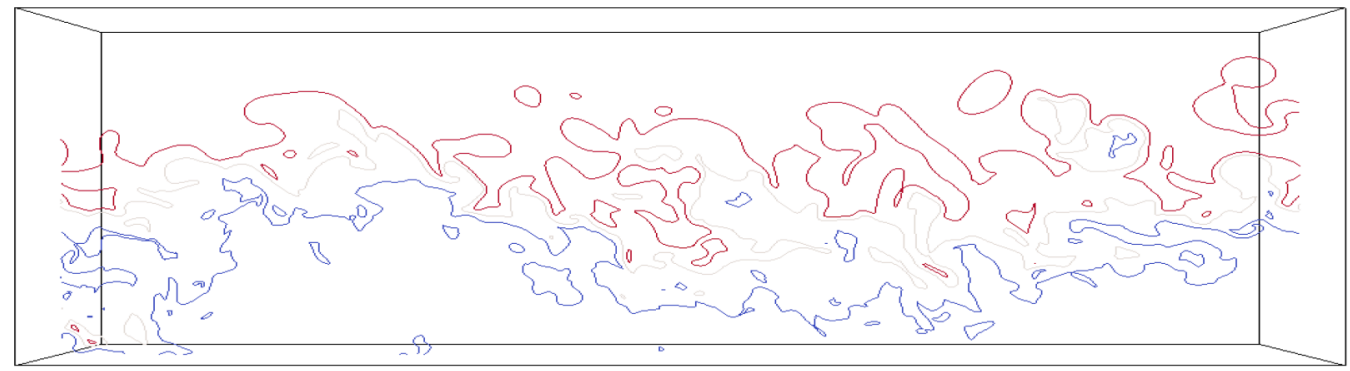

(b) Da-

FIG. 5. Contour slices of subsets of the $\mathrm{Da}+$ (top) and $\mathrm{Da}-$ (bottom) cases showing various isosurfaces of progress variable $c$ ranging from the leading edge represented by blue lines to the trailing edge represented by red lines.

from the top to the bottom row. The total number of interactions observed is of the order 10000 for $u^{\prime}=10 s_{L}, 40000$ for $u^{\prime}=20 s_{L}$, and 200000 for $u^{\prime}=40 s_{L}$. This is consistent with the findings of Trivedi et al. [23].

It is also worth noting that PP and RP are spherical events whereas TC and TF are cylindrical events. It can been seen in the histograms that the cylindrical topological events (sum of TC and TF) occur more frequently than the spherical events (sum of RP and PP). This is consistent with the findings of Pope et al. [53] and Cant et al. [54].

The corresponding flame-flame interaction statistics evaluated for the Hawkes et al. [43] dataset are presented in Fig. 7 for both $\mathrm{Da}+$ (top) and $\mathrm{Da}-$ (bottom) cases. Note that the reaction progress variable is defined here based on hydrogen mass fraction. Only a single snapshot is analyzed for each case. Different colors show the different types of topologies (see legend). The general shape of the histograms differs for the two cases but shows a common trend. Both cases show a significant number of critical points, and hence flame-flame interactions, at the leading edge of the flame $(0.01<c<0.3)$, but their frequency of occurrence decreases in the middle of the flame $(0.3<c<0.9)$. Then, there is a resurgence of the number of flame-flame interactions toward the trailing edge $(c>0.9)$.

These histograms are similar to those presented by Griffiths et al. [31] with two exceptions: (1) a progress variable based on $\mathrm{H}_{2}$ instead of $\mathrm{H}_{2} \mathrm{O}$ is used in the analysis to provide a better representation of the reaction zone within the flame, and (2) a smaller subset of the Hawkes et al. 

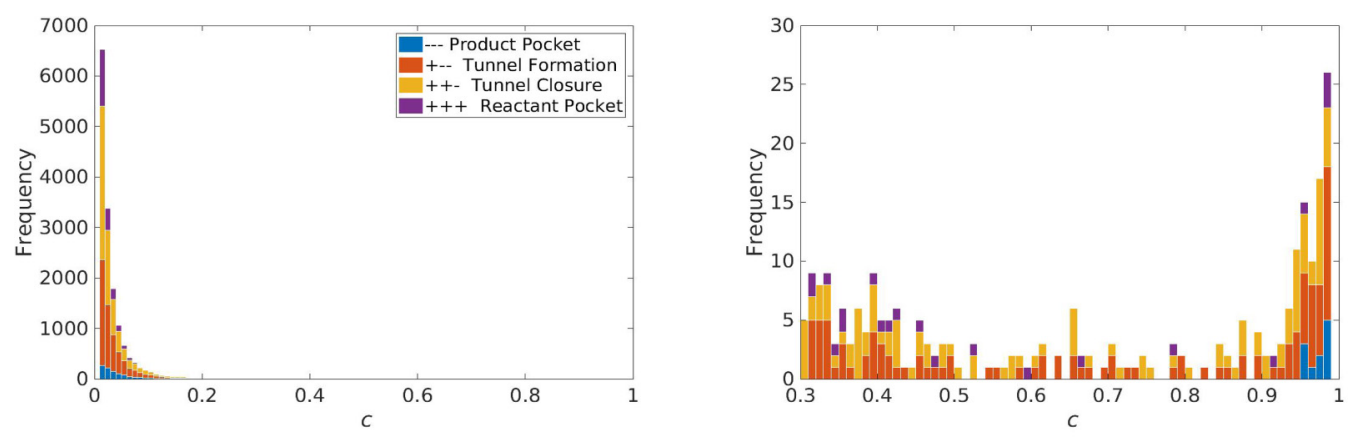

(a) $u^{\prime} / s_{L}=10$
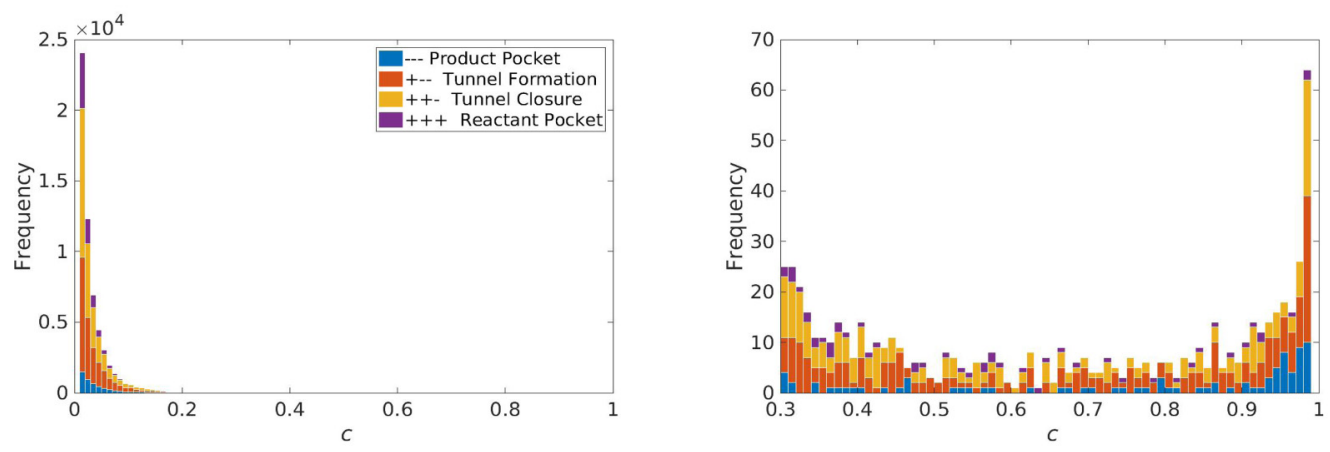

(b) $u^{\prime} / s_{L}=20$
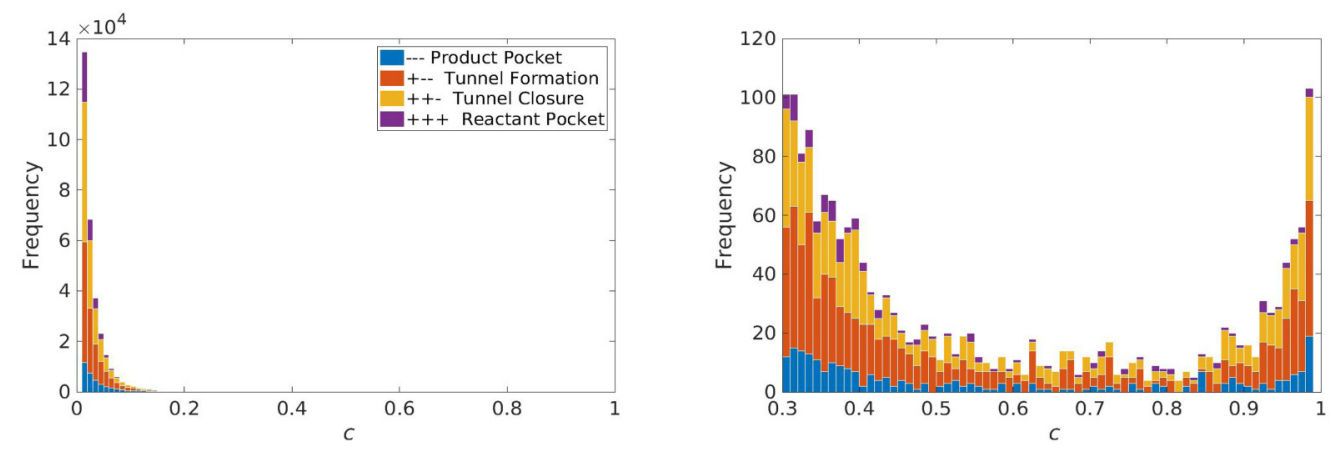

(c) $u^{\prime} / s_{L}=40$

FIG. 6. Histograms for the frequency of occurrence of different topologies in the hydrocarbon-air dataset represented by different colors. The left column shows the results for $0.01<c<0.99$, whereas the right column shows the results for $0.3<c<0.99$. The turbulence intensity is increased from $u^{\prime}=10 s_{L}$ (top row) to $u^{\prime}=20 s_{L}$ (middle row) to $u^{\prime}=40 s_{L}$ (bottom row).

dataset [43] was used. However, the sample size is sufficiently large to provide the same general shape of the histograms as in Ref. [31].

It should be noted that the domain size for the $\mathrm{Da}-$ case is half the length in all directions and hence contains a smaller flame area than the $\mathrm{Da}+$ case. Therefore the cross-sectional area in these two cases differs by a factor of four and hence the statistics cannot be directly compared. To make 

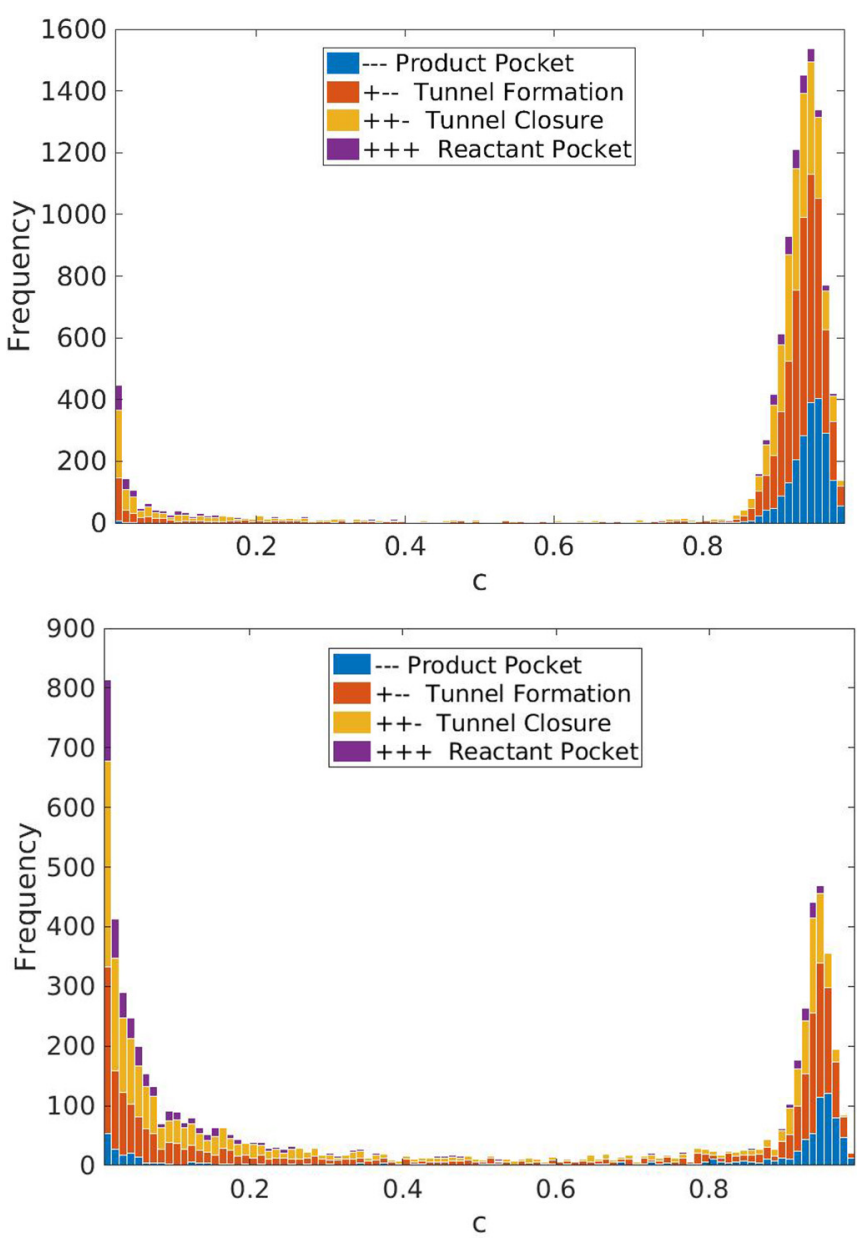

FIG. 7. Histograms showing the number of flame-flame interactions in the hydrogen-air dataset for Da+ (top) and $\mathrm{Da}-$ (bottom) cases.

a direct comparison between these cases, the number of interactions is divided by the surface area of the isosurface at $c=0.5$ and the results are presented in Fig. 8 .

It is evident from Fig. 8 that at the trailing edge, the number of flame-flame interactions per unit surface area is similar in magnitude for both cases $\left(\sim 6 \times 10^{5}\right.$ for the Da+ case and $\sim 8 \times 10^{5}$ for the $\mathrm{Da}-$ case). By contrast, the number of interactions is larger in magnitude at the leading edge for the $\mathrm{Da}-$ case. The reasons for the observed shapes of the histograms as well as the similarities and differences between the $\mathrm{Da}+$ and $\mathrm{Da}-$ cases will be explained in more detail below. These results will also be used to bring out the effects of turbulence intensity, turbulence length scales and thermodiffusive phenomena more clearly.

The difference between the critical point statistics of hydrocarbon flames and hydrogen flames is quite apparent by comparing Figs. 6 and 8. Note that the turbulence intensities and length scales of the two datasets are not identical and hence they cannot be compared directly. The aim here is to analyze the relative occurrence of the various flame-flame interaction types for each case, and to elucidate the reasons for the observed trends.

In the hydrocarbon flames, the number of interactions toward the leading edge of the flame, i.e., $c<0.3$ [Fig. 6 (top)] is much greater than for the rest of the flame [Fig. 6 (bottom)]. There is an increase in the number of interactions observed toward the trailing edge of the flame compared 

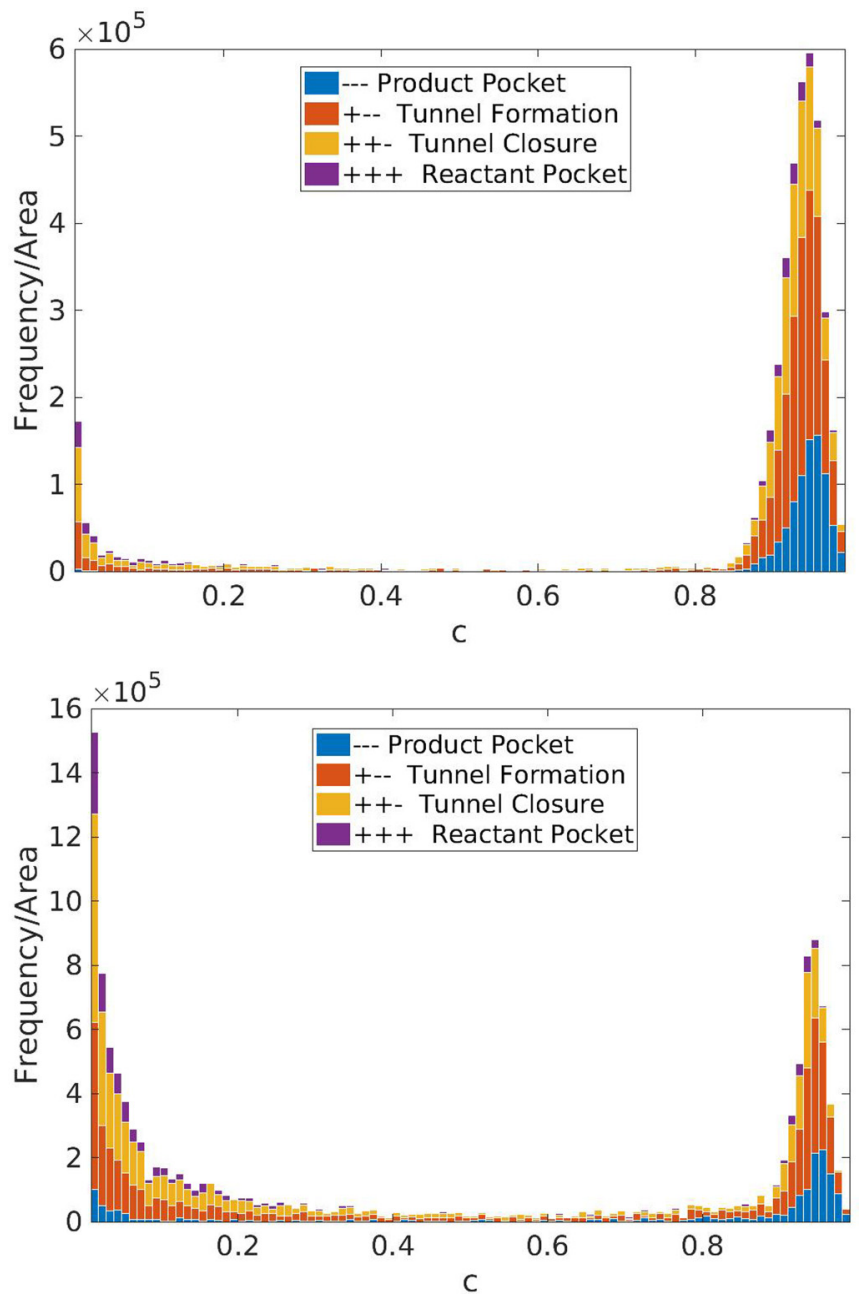

FIG. 8. Histograms showing the number of flame-flame interactions normalized by the surface area in the hydrogen-air dataset at $c=0.5$ for $\mathrm{Da}+$ (top) and $\mathrm{Da}-$ (bottom) cases.

to the middle part of the flame as seen in the right-hand column of Fig. 6, but the number of interactions at the trailing edge still remains well below the number of interactions at the leading edge for all three turbulence-intensity cases. In the hydrogen flames, the number of flame-flame interactions observed at the trailing edge is significantly higher than for the hydrocarbon flames. The ratio of leading edge interactions to trailing edge interactions is larger for the $\mathrm{Da}-$ case than for the $\mathrm{Da}+$ case. In all cases, for both hydrocarbon and hydrogen flames, very few interactions occur in the middle of the flame.

\section{A. Interactions in hydrocarbon flames}

The flame-flame interaction pattern of the hydrocarbon flames is discussed first. The decrease in the number of flame-flame interactions with increasing values of $c$ can be attributed to the dissipation of turbulence within the flame. The temperature rises across the flame as $c$ increases and, hence, the viscosity increases and results in an increase in the viscous dissipation of turbulence. In the absence of a source of turbulence within the flame, the turbulence monotonically decays within the flame and as a result, the number of flame-flame interactions decreases with increasing $c$ values. The effects of 


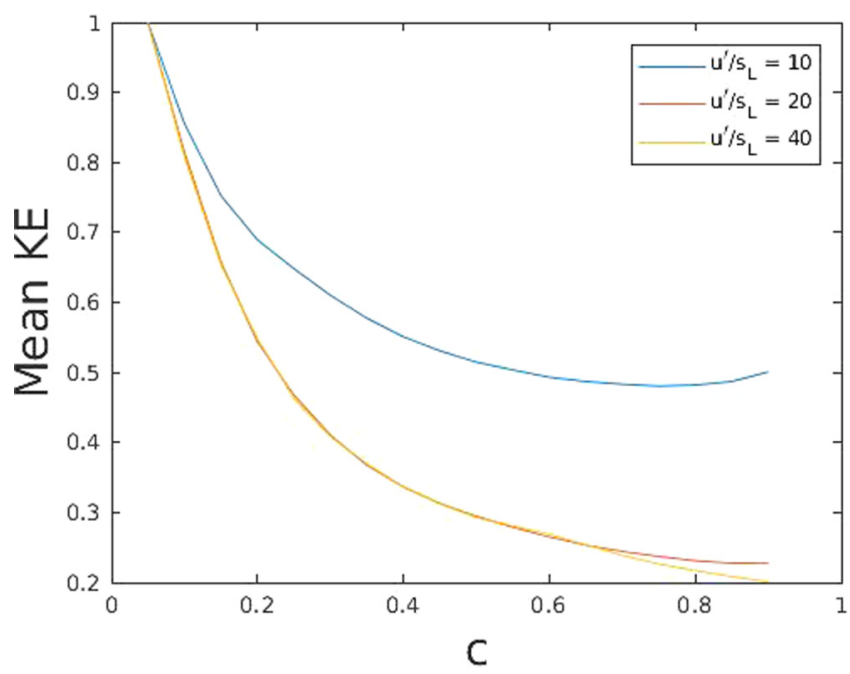

FIG. 9. Mean turbulent kinetic energy averaged over surfaces of progress variable $c$ for the hydrocarbon flames and normalized by its values in the unburnt mixture. The KE decays as $c$ increases through the flame.

dilatation due to heat release have been considered in previous studies [55] and found to have little influence on the rate of decay.

Figure 9 shows the turbulent kinetic energy per unit volume averaged over surfaces of progress variable $c$ plotted for the hydrocarbon flames after $t=4 \tau$. The turbulent kinetic energy is normalized by its value in the unburnt mixture and can be seen to decay as $c$ increases through the flame.

The turbulence in the fresh reactants also decays with time and therefore the interaction statistics taken at individual snapshots in time are different from each other. Nevertheless the general shape of the histogram is very similar for every snapshot. Hence the time-averaged statistics presented here do provide a good representation of that shape. Future work will make use of forced turbulence $[56,57]$ between the two interacting flames to provide a statistically stationary value of the turbulent kinetic energy.

Figure 10(a) shows the flame behavior qualitatively for the $u^{\prime}=20 s_{L}$ case. In this figure, the blue surface is the isosurface at $c=0.1$ representing the leading edge of the flame and the red surface an isosurface at $c=0.9$ representing the trailing edge of the flame. It can be observed from this figure that the blue surface exhibits numerous high curvature cusps. On the other hand, the red surface exhibits fewer cusps and these are of lower curvature. To quantify this behavior, it is helpful to examine the flame curvature.

The mean of the two principal curvatures $h_{1}$ and $h_{2}$ of a three-dimensional flame surface can be evaluated locally as

$$
\kappa=\frac{1}{2}\left(h_{1}+h_{2}\right)=\frac{1}{2} \frac{\partial n_{i}}{\partial x_{i}},
$$

where $n_{i}$ is the local flame normal vector. The curvature is considered positive when the flame surface is convex toward the reactants and vice versa. For a statistically planar flame, the mean curvature of the flame surface is equally likely to be positive or negative, and therefore the curvature is zero when averaged over a flame surface. The absolute value of mean curvature $|\kappa|$, however, gives a measure of the curvature of the surface regardless of its direction. This quantity averaged over isosurfaces of $c$ is plotted against $c$ in Fig. 10(b), normalized by the unstrained laminar flame thickness $\delta_{L}$. This figure represents the extent of wrinkling at different isosurfaces across the flame. The blue, red, and yellow curves in the figure show values of averaged absolute mean curvature $\overline{|\kappa|}$ for the $u^{\prime}=10 s_{L}, 20 s_{L}$, and $40 s_{L}$ cases, respectively. It is clear in Fig. 10(b) that $\overline{|\kappa|}$ is highest 


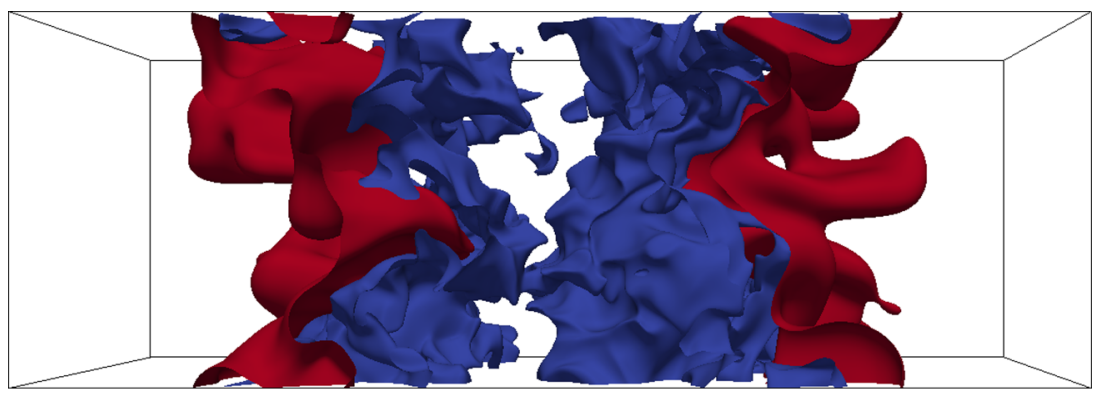

(a)

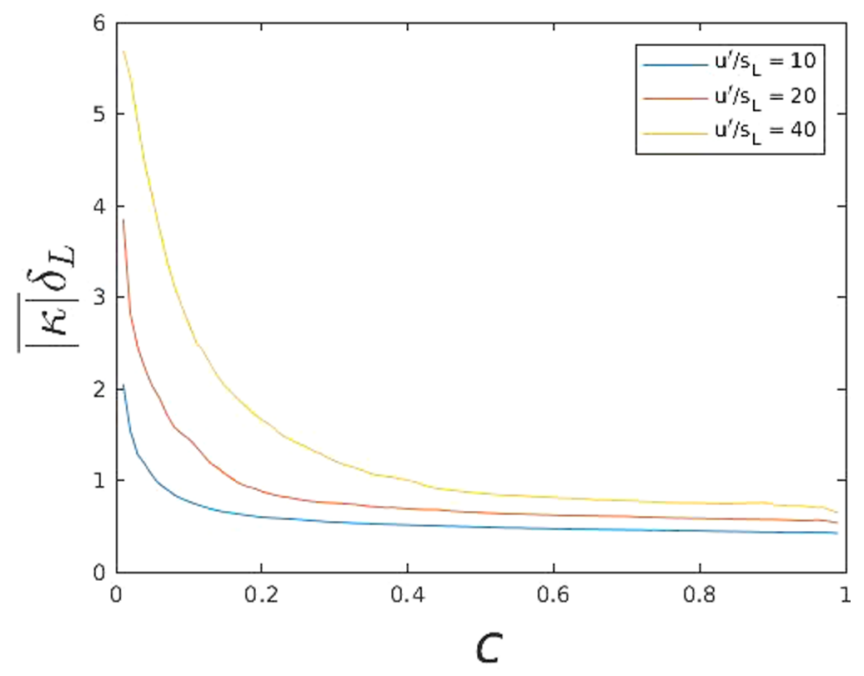

(b)

FIG. 10. (a) Contours of progress variable at $c=0.1$ representing the leading edge (blue) and at $c=0.9$ representing the trailing edge (red) of the flame; (b) absolute value of the mean curvature surface averaged over the isosurfaces of progress variable $c$ normalized by unstrained laminar flame thickness $\delta_{L}$, plotted against $c$ for the hydrocarbon flame cases.

for the lowest value of $c$. The isosurface is therefore more wrinkled at lower values of $c$ and hence results in a high number of flame-flame interactions. As $c$ increases, the turbulence decays within the flame and the value of $\overline{|\kappa|}$ decreases monotonically. Consequently, the number of flame-flame interactions also decrease as $c$ increases.

The surface averaged value of mean curvature $\overline{|\kappa|}$ is not the only marker for the occurrence of flame-flame interactions. On close inspection of Fig. 10(b), it can be observed that the value of $\overline{|\kappa|}$ at $c=0.3$ for $u^{\prime}=40 s_{L}$ is similar to that at $c=0.1$ for $u^{\prime}=20 s_{L}$. However, the number of flame-flame interactions corresponding to these values are 100 and 600, respectively. Likewise, the value of $\overline{|\kappa|}$ at $c=0.3$ for $u^{\prime}=20 s_{L}$ is identical to $\overline{|\kappa|}$ at $c=0.10$ for $u^{\prime}=10 s_{L}$, but the number of interactions at these values are 25 and 200, respectively. This indicates that the location of the flame at which the flame-flame interactions are evaluated also has an important effect on their statistics. The explanation for this behavior is provided below.

The reaction rate profile for a one-dimensional unstrained laminar flame simulated with singlestep chemistry is plotted against the progress variable in Fig. 11. All parameters for this 1D flame are exactly the same as the initial laminar flame profile used in the twin flame dataset. The reaction 


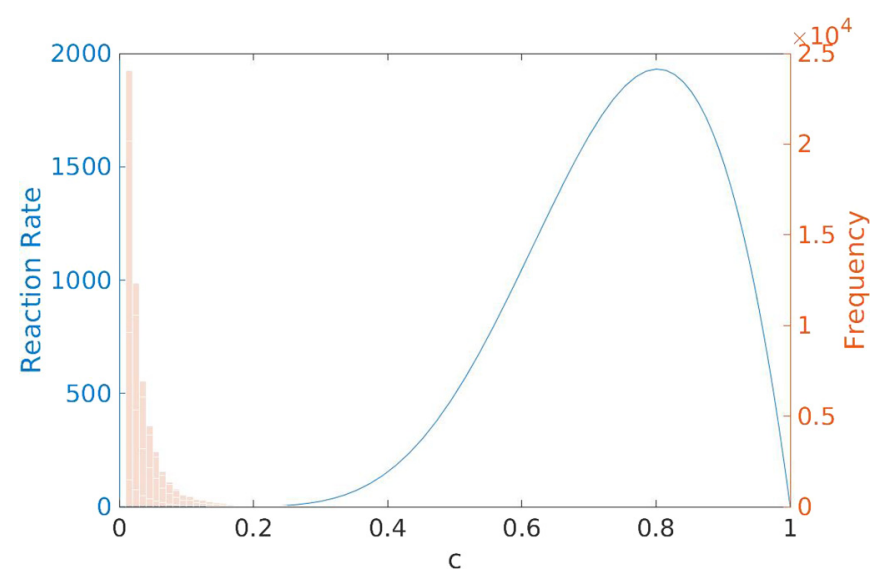

FIG. 11. Reaction rate for a 1D laminar hydrocarbon flame plotted against progress variable $c$ showing the rise in reaction rates and the subsequent drop in the number of interactions represented by the shaded background.

rate is plotted after the 1D flame reaches a steady state. Figure 11 shows that the reaction rate (blue line) becomes nonzero at $c \simeq 0.3$ and with a peak value at around $c=0.8$. The shaded background in this figure represents the general shape of the histogram for the $u^{\prime}=20 s_{L}$ case. Note that the flame-flame interactions disappear in the region $0.3<c<0.9$ where the reaction rate is significant.

The rise in the rate of reaction causes enhanced gradients of both the temperature and progress variable. In physical space, this manifests itself by causing the isosurfaces of the progress variable to come very close to each other. This can be observed in Fig. 12 in which a slice of a subsection of the $u^{\prime}=20 s_{L}$ case is shown. For simplicity, a subsection is carefully chosen such that it contains only one flame and shows the self-interactions within this flame. The white lines in each subfigure represent a pair of isocontours of $c$. In Fig. 12(a), the isocontours are at $c=0.05$ and 0.07, Fig. 12(b) shows isocontours at $(c=0.20$ and 0.22$)$, Fig. 12(c) shows isocontours at $c=0.50$ and 0.52 and Fig. 12(d) shows isocontours at $c=0.90$ and 0.92. Therefore, each pair is $c=0.02$ apart and represents the leading edge, the approximate point at which reactions begin, middle, and the trailing edge of the flame, respectively. The distance between the pair of isocontours at the leading edge [Fig. 12(a)] is large but it decreases as the reactions begin [Fig. 12(b)]. In the middle of the flame, the isocontours become nearly inseparable [Fig. 12(c)].

This has an important effect on the flame-flame interaction statistics. Recall that for flames in the thin reaction zones regime, i.e., all the flames analyzed in this work, the isosurfaces of progress variable are not always parallel to each other. They can bend individually and cause self interactions. The relatively large separation between isocontours in Fig. 12(a) allows them to curve and selfinteract under the influence of turbulence and flame propagation, whereas the small separation between the isocontours in the middle does not offer enough room for significant local curvature of any individual isocontour. This, combined with the decrease in the absolute mean curvature of the flame, explains why the flame-flame interactions are less frequent in the middle of the flame.

At the trailing edge of the flame, the separation between the isocontours is larger compared to that in the middle of the flame [Fig. 12(d)]. This provides more room for the self-interactions despite the fact that the surface averaged absolute value of mean curvature $\overline{|\kappa|}$ is the lowest at the trailing edge of the flame [see Fig. 10(b)]. Therefore, the number of flame-flame interactions shows a slight increase at the trailing edge of the flame as seen in the right-hand side subfigures in Fig. 6.

\section{B. Interactions in hydrogen flames}

For hydrogen flames, the picture is somewhat different. Figure 13 shows the graph of the absolute value of mean curvature normalized by the unstrained laminar flame thickness $\delta_{L}$ plotted against 


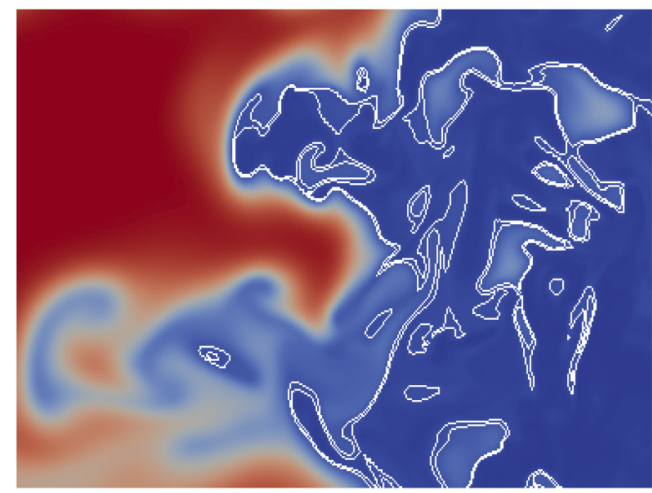

(a) Iso-contours at $c=0.05$ and 0.07

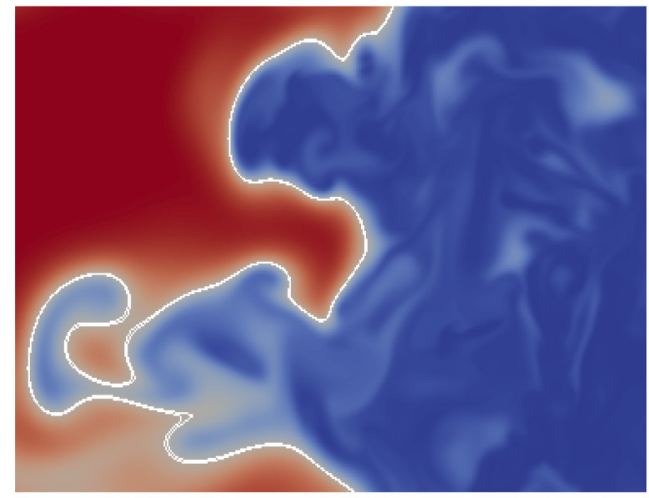

(c) Iso-contours at $c=0.50$ and 0.52

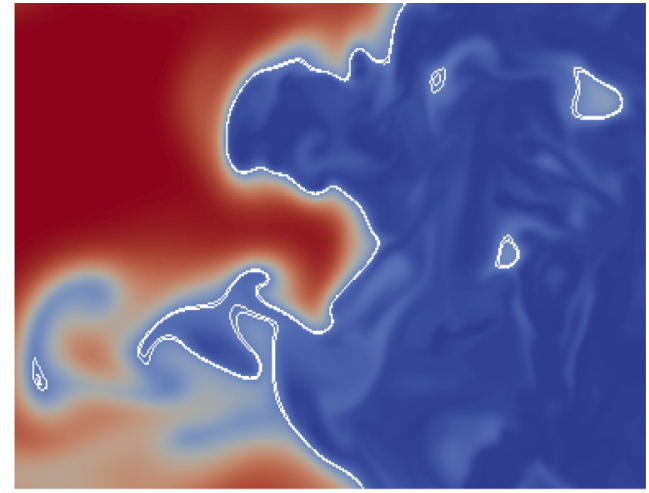

(b) Iso-contours at $c=0.20$ and 0.22



(d) Iso-contours at $c=0.90$ and 0.92

FIG. 12. Slices of the mass fraction profile showing a pair of isosurfaces separated by $c=0.02$ at four different locations of the flame for $u^{\prime}=20 s_{L}$ case.

progress variable for the $\mathrm{Da}+$ (red line) and $\mathrm{Da}-$ (blue line) cases. At the leading edge $(c<0.1)$, the absolute mean curvature for the $\mathrm{Da}-$ flame is higher than that for the $\mathrm{Da}+$ flame. This is because of the higher turbulence intensity (see Sec. III) for the $\mathrm{Da}-$ case which results in relatively more flame-flame interactions at the leading edge.

However, at the trailing edge, both cases show a rise in the absolute mean value of curvature despite the dissipation of turbulence caused by the increase in viscosity brought on by the higher temperature. The rise of absolute mean curvature is more pronounced for the $\mathrm{Da}+$ case than for the $\mathrm{Da}-$ case but the absolute mean curvature value is roughly the same.

There are two reasons for this. First, the hydrogen-air flame in the dataset used above interacts with shear generated turbulence. Chaudhari et al. [52] plotted the fractional contribution of mean shear with the total strain and found that the effect of mean shear was stronger at the trailing edge of the flame for both $\mathrm{Da}+$ and $\mathrm{Da}-$ cases.

The second reason for the increased number of flame-flame interactions is the effect of thermodiffusive instabilities at the low Lewis numbers experienced in the hydrogen flames. These instabilities are enhanced toward the trailing edge of the flame where the Lewis number takes its lowest value. This can be shown by analyzing the changes in Lewis number in a one-dimensional hydrogen-air laminar flame simulated using the code Senga2 [49]. This flame was simulated in a $1 \mathrm{D}$ inflow-outflow domain of length $1.5 \mathrm{~cm}$ in the $x$ direction, which corresponds to $30 \delta_{L}$. A 


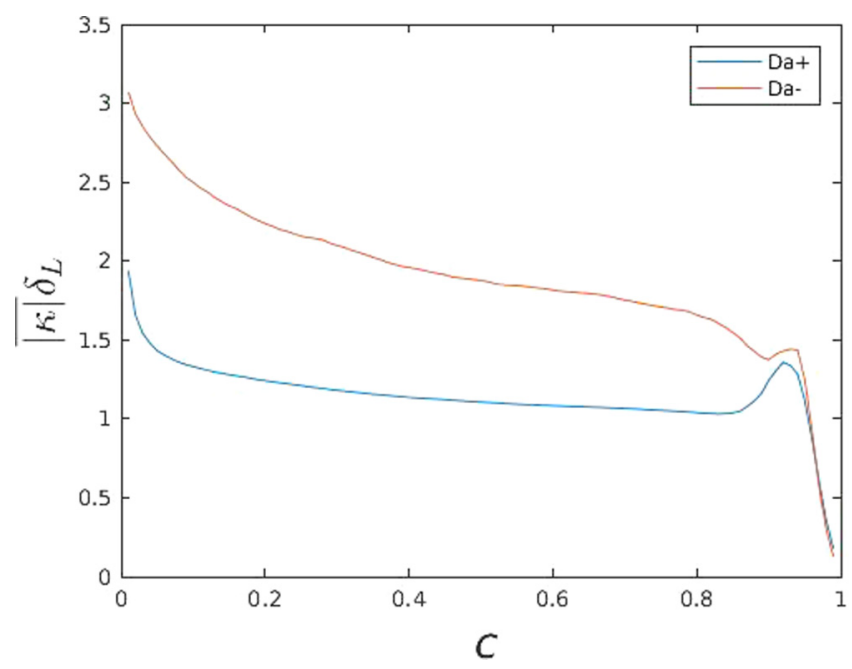

FIG. 13. Surface averaged absolute value of the mean curvature $\overline{|\kappa|}$ normalized by the unstrained laminar flame thickness $\delta_{L}$, plotted against the progress variable $c$ for the hydrogen-air dataset. In this figure, the blue line represents $\overline{|\kappa|}$ for the $\mathrm{Da}+$ case and the red line represents $\overline{|\kappa|}$ for the $\mathrm{Da}-$ case.

nine-species, 21-step mechanism [50] was used with the equivalence ratio of the mixture set to $\phi=0.7$ and the reactants preheated to $700 \mathrm{~K}$. This made the initial mixture the same as that used in the Hawkes et al. dataset [43]. The unstrained laminar flame speed and flame thickness for this mixture are $7.9 \mathrm{~m} / \mathrm{s}$ and $0.5 \mathrm{~mm}$, respectively. The reactants enter the domain with an inflow velocity of $7.9 \mathrm{~m} / \mathrm{s}$ to match the flame speed of the mixture and keep the flame stationary in space.

The thermal conductivity $\lambda$ for the mixture and mass diffusivity $D$ of $\mathrm{H}_{2}$ are calculated using the mixture averaged transport approach. Using these values, the Lewis number is evaluated and plotted against the progress variable $c$. The result obtained for this flame is shown in Fig. 14 which clearly shows that the Lewis number decreases across the flame as $c$ increases and is lowest at the trailing edge of the flame. This indicates that the thermodiffusive instabilities are further exaggerated toward

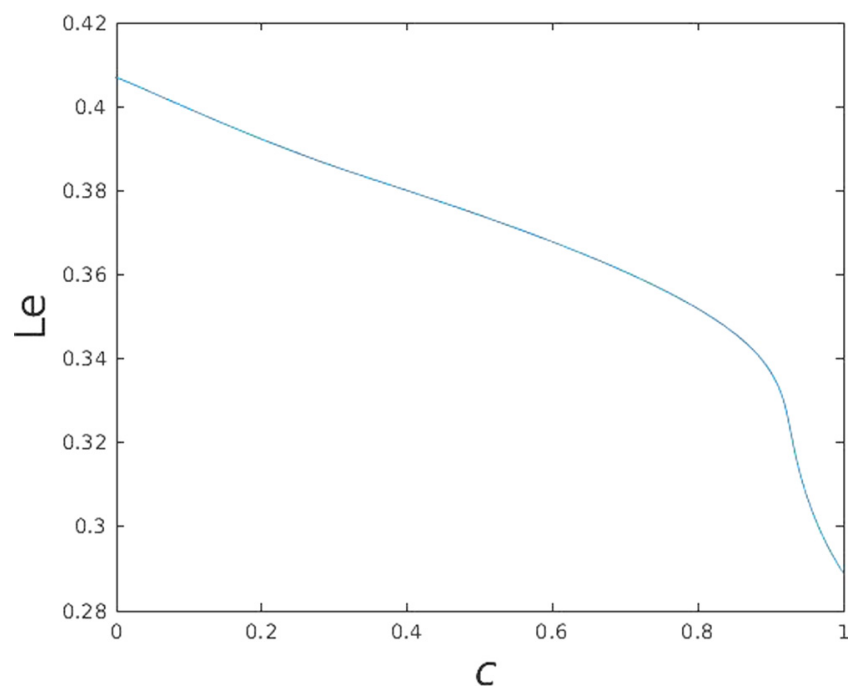

FIG. 14. Variation of Lewis number with $c$ in a 1D laminar hydrogen flame. 




(a)

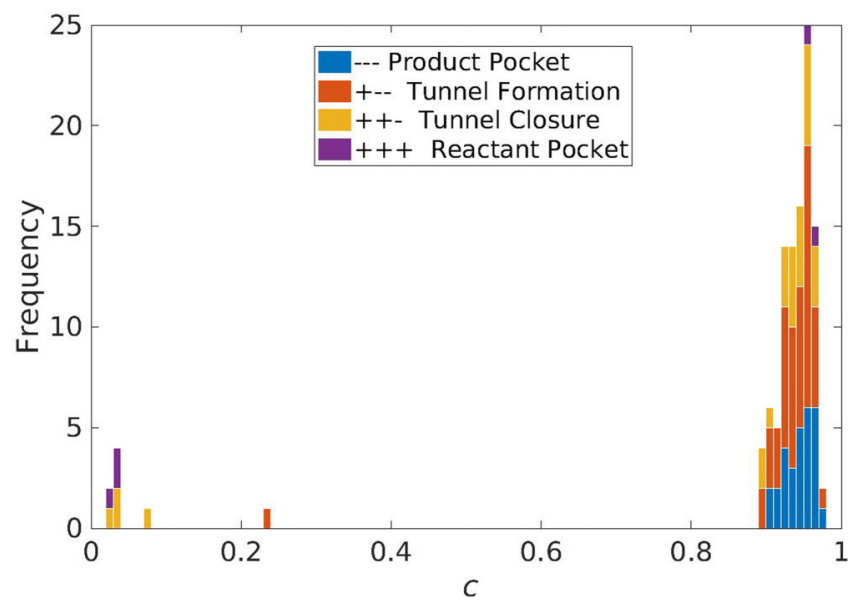

(b)

FIG. 15. Results showing the effects of thermodiffusive instabilities caused by Le $<1$ on the statistics of flame-flame interactions. (a) Surface averaged absolute mean curvature $\overline{|\kappa|}$ normalized by the unstrained laminar flame thickness $\delta_{L}$ plotted against $c$ for three-dimensional hydrogen-air flame in low-intensity turbulence. (b) Histogram showing frequency of occurrence of flame-flame interactions for three-dimensional hydrogen-air flame in low-intensity turbulence.

the trailing edge of the flame resulting in increased flame curvature and more frequent flame-flame interactions.

The importance of Le $<1$ for flame wrinkling at the trailing edge of the flame is demonstrated further through a simulation of a lean hydrogen-air flame evolving in three-dimensional lowintensity turbulence. For this simulation, a single $1 \mathrm{D}$ laminar $\mathrm{H}_{2}$-air flame profile computed as described above was mapped on a 3D domain along with a pre-computed field of homogeneous and isotropic turbulence. The intensity of turbulence was kept low so as to trigger thermodiffusive instabilities without causing flame-flame interactions directly. Inflow-outflow boundary conditions were employed with reactants incoming at the laminar flame speed $(=7.9 \mathrm{~m} / \mathrm{s})$. The flame was allowed to interact with the turbulence for $t=2.5 \tau$ and the statistics of flame-flame interactions were recorded [31]. The results for this flame are shown in Fig. 15. 


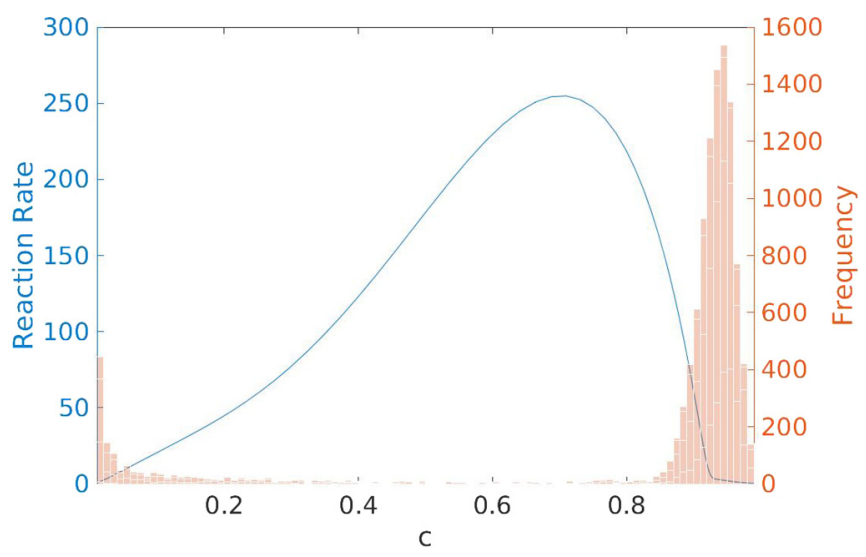

FIG. 16. Reaction rate profile for a 1D laminar hydrogen flame (blue line) plotted against $c$. The shaded red background represents the general shape of the histograms of frequency of flame-flame interactions for the $\mathrm{Da}+$ case.

It can be seen in Fig. 15(a) that there is a significant rise in $\overline{|\kappa|}$ at the trailing edge of the flame consistent with the results for the $\mathrm{Da}+$ and $\mathrm{Da}-$ cases. Keeping in mind that there is no turbulence generation in this case and that the homogeneous isotropic turbulence decays over time, the increase in $\overline{|\kappa|}$ observed at the trailing edge of the flame arises only from the action of the thermodiffusive instabilities due to Le $<1$. The resulting histogram of the frequency of flame-flame interactions is shown in Fig. 15(b). Very few flame-flame interactions are observed at the leading edge because of the low intensity of turbulence. However, a significant number of flame-flame interactions occurs at the trailing edge. It is therefore clear that a significant fraction of the trailing edge flame-flame interactions observed for the hydrogen-air flames results from the nonunity Lewis number effects on the thermodiffusive balance of the flame.

The combined effect of mean shear in the hydrogen flame dataset and the thermodiffusive instabilities explains the peak in $\overline{|\kappa|}$ and the corresponding increase in the flame-flame interactions at the trailing edge of the flame. In the middle of the hydrogen air flames $(0.3<c<0.9)$, the frequency of interactions decreases considerably, similar to what was observed for the hydrocarbon flames. This is attributed to the high gradients of temperature and species mass fraction caused by the increase in reaction rate as explained above. Figure 16 shows the reaction rate (blue line) plotted against $c$ for a 1D unstrained laminar hydrogen-air flame with the same chemistry and initial parameters as in the Hawkes et al. [43] dataset. The shaded background shows the shape of the histograms of the number of flame-flame interactions for the $\mathrm{Da}+$ case. The number of flame-flame interactions can be seen to diminish as the reaction rate becomes significant. Also note that the number of flame-flame interactions toward the trailing edge becomes significant as the reaction rate decreases.

\section{Twin hydrocarbon flames vs. single hydrocarbon flame}

It is interesting to look at the fraction of the individual flame topologies as the turbulence intensity is increased in the twin hydrocarbon flame configuration. Figure 17 (top) shows that the fraction of product pockets increases with the turbulence intensity, while the fraction of reactant pockets decreases. The tunnel formation and tunnel closure events exhibit similar behavior in that the fraction of tunnel formation increases and the corresponding fraction of tunnel closure decreases as turbulence intensity increases [Fig. 17 (bottom)]. These fractions are also presented in Table II. Note that the fraction of cylindrical topologies (TC and TF) dominates over that of spherical topologies (RR and RP), in agreement with previous results [53,54]. 


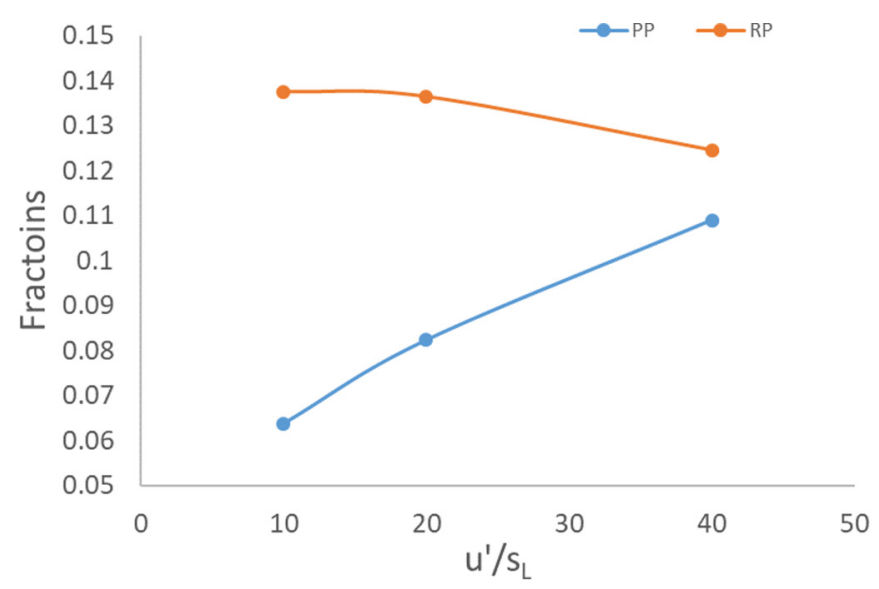

(a)

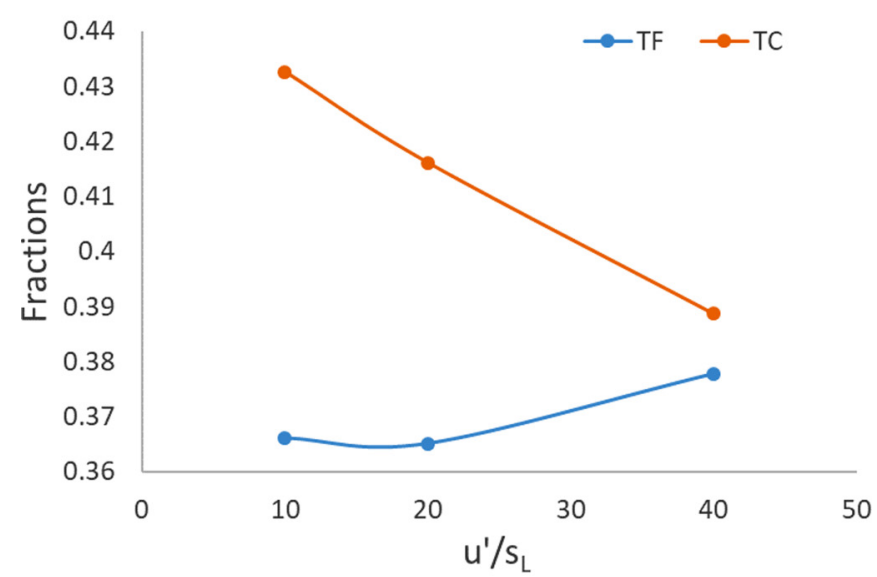

(b)

FIG. 17. Fractions of RP and PP (top) and fractions of TF and TC (bottom) for the twin flame dataset.

This result is also consistent with the previous findings of Trivedi et al. [23] in which the statistics of single hydrocarbon flames were extracted. The key difference is that previously the simulations for different values of $u^{\prime}$ were run for the same number of eddy turnover times whereas in this current analysis, the flames are simulated until the two flames fully interact with each other. The increase in reactant pockets and decrease in product pockets with increasing turbulence level was also observed experimentally in a recent publication by Tyagi et al. [58].

TABLE II. Fraction of topologies for increasing turbulent intensities for the twin hydrocarbon flame configuration.

\begin{tabular}{lcccr}
\hline \hline$u^{\prime} / s_{L}$ & PP & TF & TC & RP \\
\hline 10 & 0.099 & 0.420 & 0.389 & 0.091 \\
20 & 0.118 & 0.425 & 0.369 & 0.089 \\
40 & 0.150 & 0.427 & 0.346 & 0.082 \\
\hline \hline
\end{tabular}



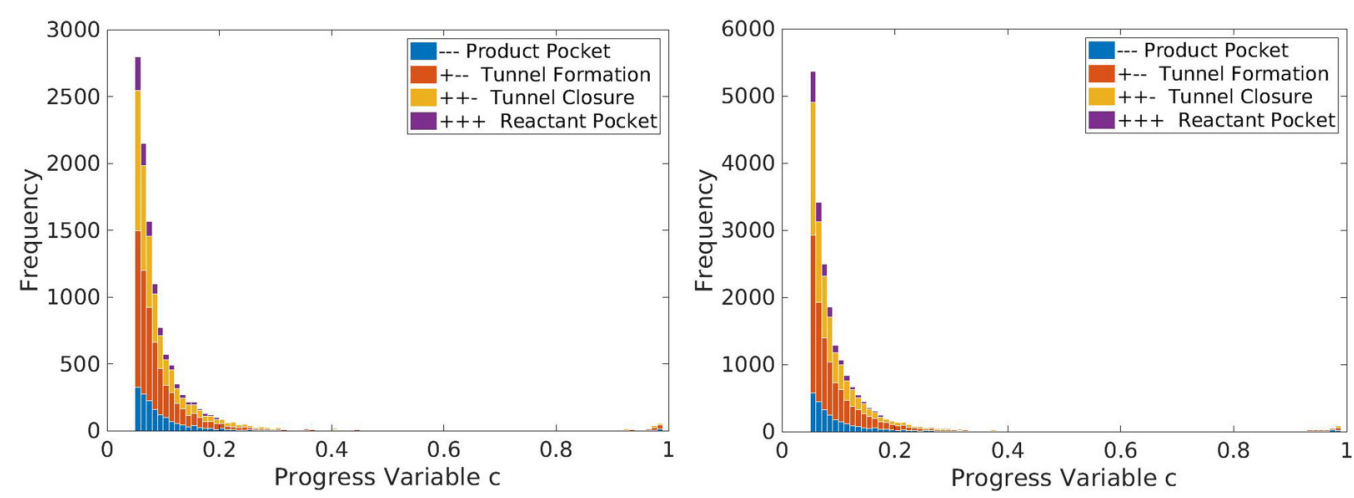

FIG. 18. Histograms showing the number of interactions in a single flame (left) vs. twin flames (right). The number of interactions for the twin flame configuration is approximately twice as that of the single flame.

Another comparison is drawn between the pair of hydrocarbon flames and a single hydrocarbon flame both simulated at turbulence intensity $u^{\prime}=20 s_{L}$. The number of interactions is recorded for the same number of eddy turnover times in each case. The single flame freely propagates in the domain, whereas the flames in the twin flame configuration will ultimately interact with each other. The results for the frequency of occurrence of the different topologies are shown in Fig. 18.

It is found that the number of interactions in the case of two flames is nearly twice as large as the number of interactions observed for a single flame. This is an important result since it indicates that having two flames does not significantly increase the probability of capturing the flame-flame interactions.

\section{CONCLUSIONS}

The analysis for hydrocarbon flames indicates that the histograms of frequency of occurrence of the flame-flame interactions clearly show that more interactions are found for higher turbulence intensities. Most of the flame-flame interactions are found at the leading edge and are infrequent at the trailing edge.

For hydrogen-air flames, a somewhat different picture of the flame-flame interactions was observed in which a significant number of interactions was found at the trailing edge of the flame. This is attributed to two reasons: (1) The fraction of mean shear over total strain was found to increase at the trailing edge of hydrogen-air flames [52], and (2) the thermodiffusive instabilities at low Lewis number are more prominent at the trailing edge of the flame. These effects resulted in a net increase in absolute mean curvature of the flame at the trailing edge causing numerous flame-flame interactions in that region. Very few interactions were found in the middle region for both hydrocarbon and hydrogen flames where chemical reactions occur.

The proportion of different topologies varies with the turbulence intensity for the twin hydrocarbon flame configuration. There is an increase in product pockets and tunnel formation events with increasing turbulence intensity from $u^{\prime} / s_{L}=10$ to $u^{\prime} / s_{L}=40$, and a corresponding decrease in reactant pockets and tunnel closure events for those intensities. This is consistent with previous findings [23] in which only self interactions in a single flame were considered. The above result may have implications for flame propagation with increasing turbulence intensity. Finally, it was found that the number of interactions in the case of two flames is nearly twice as much as that with a single flame. This indicates that most of the observed flame-flame interactions are self-interactions within each flame, and proportionately few interactions occur due to flame collision.

The reactant pocket and tunnel closure topologies result in a very high displacement speed near burnout $[19,33,38]$. The increase in burning rate due to reactant pockets was also observed in a dual burner flame experiment [58]. The decrease in the proportions of these topologies indicates that 
the overall flame speed might be reduced at higher turbulence intensities. Future work will involve investigating individual topologies and their specific role in flame propagation. Examples of the individual topologies have already been captured in subdomains that can be extracted and simulated separately. Their local effects can then be combined with the flame-flame interaction statistics to predict their effect on overall flame properties.

The results from the simplified single-step reaction mechanism need to be validated using more detailed hydrocarbon reaction schemes. Parametric studies involving systematic changes in the Lewis number need to be performed and validated against the existing results [22]. Flame-flame interactions in different fuel types need to be compared. The results from the critical point method of finding flame-flame interactions [31] needs to be compared with other such techniques, for example the the wavelet transform method used by Dunstan et al. [21]. The numerical results should also be compared against experimental data obtained specifically for flame-flame interactions $[44,45]$. Further comparisons between the present results and those for high-intensity turbulence [46,59] will also be useful. The goal is to develop robust modeling that can account for all the significant changes in flame properties that may occur due to flame-flame interaction.

\section{ACKNOWLEDGMENTS}

This work was funded under the embedded CSE programme of the ARCHER UK National Supercomputing Service. The Cambridge University authors are grateful for the financial support from the Cambridge Trust and the Nehru Trust. The work at Sandia National Laboratories was supported by the U.S. Department of Energy, Office of Basic Energy Sciences, Division of Chemical Sciences, Geosciences, and Biosciences. Sandia National Laboratories is a multimission laboratory managed and operated by National Technology and Engineering Solutions of Sandia, LLC, a wholly owned subsidiary of Honeywell International, Inc., for the U.S. Department of Energy's National Nuclear Security Administration under Contract No. DE-NA-0003525. Special thanks are due to Ryan Griffiths for providing the numerical tools and for valuable discussions on this topic.

[1] B. Karlovitz, D. W. Denniston, and F. E. Wells, Investigation of turbulent flames, J. Chem. Phys. 19, 541 (1951).

[2] A. M. Klimov, Laminar flame in turbulent flow, Prikladnoy Mekhaniki i Tekhnicheskoy Fiziki Zhurnal (English translation AD-A200 241 Foreign Technology Division, Air Force Systems Command, 1988), 3, 49 (1963).

[3] F. A. Williams, Combustion Theory (Westview Press, Boulder, CO, 1985).

[4] C. K. Law, Dynamics of stretched flames, Symposium (International) on Combustion 22, 1381 (1989).

[5] D. Bradley, How fast can we burn?, in Proceedings of the 24th International Symposium on Combustion (The Combustion Institute, Pittsburgh, PA, 1992), pp. 247-262.

[6] N. Peters, P. Terhoeven, J. H. Chen, and T. Echekki, Statistics of flame displacement speeds from computations of 2-D unsteady methane-air flames, in Proceedings of the 27th International Symposium on Combustion (The Combustion Institute, Pittsburgh, PA, 1998), pp. 833-839.

[7] T. Echekki and J. H. Chen, Unsteady strain rate and curvature effects in turbulent premixed methane-air flames, Combust. Flame 106, 184 (1996).

[8] N. Chakraborty and R. S. Cant, Unsteady effects of strain rate and curvature on turbulent premixed flames in an inflow-outflow configuration, Combust. Flame 137, 129 (2004).

[9] N. Peters, Laminar flamelet concepts in turbulent combustion, Proc. Combust. Inst. 21, 1231 (1986).

[10] N. Peters, A spectral closure for premixed turbulent combustion in the flamelet regime, J. Fluid Mech. 242, 611 (1992).

[11] S. B. Pope, The evolution of surfaces in turbulence, Int. J. Eng. Sci. 26, 445 (1988).

[12] S. M. Candel and T. J. Poinsot, Flame stretch and the balance equation for the flame area, Combust. Sci. Technol. 70, 1 (1990). 
[13] R. S. Cant, S. B. Pope, and K. N. C. Bray, Modelling of flamelet surface-to-volume ratio in turbulent premixed combustion, in Proceedings of the 23rd International Symposium on Combustion (The Combustion Institute, Pittsburgh, PA, 1990), pp. 809-815.

[14] K. N. C. Bray, The challenge of turbulent combustion, in Proceedings of the 26th International Symposium on Combustion (The Combustion Institute, Pittsburgh, PA, 1996), pp. 1-26.

[15] N. Fogla, F. Creta, and M. Matalon, Effect of folds and pockets on the topology and propagation of premixed turbulent flames, Combust. Flame 162, 2758 (2015).

[16] C. Dopazo, J. Martin, and J. Hierro, Local geometry of isoscalar surfaces, Phys. Rev. E 76, 056316 (2007).

[17] L. Cifuentes, C. Dopazo, J. Martin, and C. Jimenez, Local flow topologies and scalar structures in a turbulent premixed flame, Phys. Fluids 26, 065108 (2014).

[18] D. Wacks, I. Konstantinou, and N. Chakraborty, Effects of Lewis number on the statistics of the invariants of the velocity gradient tensor and local flow topologies in turbulent premixed flames, Proc. R. Soc. A 474, 20170706 (2018).

[19] J. H. Chen, T. Echekki, and W. Kollman, The mechanism of two-dimensional pocket formation in lean premixed methane-air flames with implications to turbulent combustion, Combust. Flame 116, 15 (1999).

[20] A. Y. Poludnenko and E. S. Oran, The interaction of high-speed turbulence with flames: Turbulent flame speed, Combust. Flame 158, 301 (2011).

[21] T. D. Dunstan, N. Swaminathan, K. N. C. Bray, and N. G. Kingsbury, Flame interactions in turbulent premixed twin V-flames, Combust. Sci. Technol. 185, 134 (2013).

[22] T. D. Dunstan, N. Swaminathan, K. N. C. Bray, and N. G. Kingsbury, The effects of non-unity Lewis numbers on turbulent premixed flame interactions in a twin V-flame configuration, Combust. Sci. Technol. 185, 874 (2013).

[23] S. Trivedi, G. V. Nivarti, and R. S. Cant, Flame self-interactions with increasing turbulence intensity, Proc. Combust. Inst. 37, 2443 (2019).

[24] C. L. Chen and S. H. Sohrab, Upstream interactions between planar symmetric laminar methane premixed flames, Combust. Flame 101, 360 (1995).

[25] T. Echekki, J. H. Chen, and I. R. Gran, The mechanism of mutual annihilation of stoichiometric premixed methane-air flames, in Proceedings of the 26th International Symposium on Combustion (The Combustion Institute, Pittsburgh, PA, 1996), p. 855.

[26] S. H. Sohrab, Z. Y. Ye, and C. K. Law, Theory of interactive combustion of counterflow premixed flames, Combust. Sci. Technol. 45, 27 (1986).

[27] P. A. Libby and F. A. Williams, Strained premixed laminar flames with two reaction zones, Combust. Sci. Technol. 37, 221 (1984).

[28] J. M. Duclos, D. Verynante, and T. J. Poinsot, A comparison of flamelet models for premixed turbulent combustion, Combust. Flame 95, 101 (1993).

[29] J. F. Driscoll, Turbulent premixed combustion: Flamelet structure and its effect on turbulent burning velocities, Prog. Energy Combust. Sci. 34, 91 (2008).

[30] A. R. Kerstein, Turbulence in combustion processes: Modeling challenges, Proc. Combust. Inst. 29, 1763 (2002).

[31] R. A. C. Griffiths, J. H. Chen, H. Kolla, R. S. Cant, and W. Kollman, Threedimensional topology of turbulent premixed flame interaction, Proc. Combust. Inst. 35, 1341 (2015).

[32] Y. B. Zel'dovich, An effect which stabilises the curved front of a laminar flame, Zhurn. Prikl. Mekh. Tekh. Fiz. 1, 102 (1966).

[33] W. Kollmann and J. H. Chen, Pocket formation and the flame surface density equation, in Proceedings of the 27th International Symposium on Combustion (The Combustion Institute, Pittsburgh, PA, 1998), pp. 927-934.

[34] H. G. Im and J. H. Chen, Preferential diffusion effects on the burning rate of interacting turbulent premixed hydrogen-air flames, Combust. Flame 131, 246 (2002).

[35] A. Y. Poludnenko and E. S. Oran, The interaction of high-speed turbulence with flames: Global properties and internal flame structure, Combust. Flame 157, 995 (2010).

[36] A. N. Lipatnikov, J. Chomiak, V. A. Sabelnikov, S. Nishiki, and T. Hasegawa, Unburned mixture fingers in premixed turbulent flames, Proc. Combust. Inst. 35, 1401 (2015). 
[37] G. I. Sivashinsky, On a converging spherical flame front, Int. J. Heat Mass Transf. 17, 1499 (1974).

[38] S. Trivedi, R. A. C. Griffiths, H. Kolla, J. H. Chen, and R. S. Cant, Topology of pocket formation in turbulent premixed flames, Proc. Combust. Inst. 37, 2619 (2019).

[39] T. Schuller, D. Durox, and S. Candel, Dynamics of and noise radiated by a perturbed impinging premixed jet flame, Combust. Flame 128, 88 (2002).

[40] M. Talei, M. J. Brear, and E. R. Hawkes, A parametric study of sound generation by premixed laminar flame annihilation, Combust. Flame 159, 757 (2012).

[41] D. Brouzet, A. Haghiri, M. Talei, and M. J. Brear, Annihilation events topology and their generated sound in turbulent premixed flames, Combust. Flame 204, 268 (2019).

[42] J. Park and T. Echekki, LES-ODT study of turbulent premixed interacting flames, Combust. Flame 159, 609 (2012).

[43] E. R. Hawkes, O. Chatakonda, H. Kolla, A. R. Kerstein, and J. H. Chen, A petascale direct numerical simulation study of the modeling of flame wrinkling for large-eddy simulations in intense turbulence, Combust. Flame 159, 2690 (2012).

[44] A. Tyagi, I. Boxx, S. Peluso, and J. O'Connor, The role of flow interaction in flame-flame interaction events in a dual burner experiment, Proc. Combust. Inst. 37, 2485 (2019).

[45] A. Tyagi, I. Boxx, S. Peluso, and J. O'Connor, Statistics and topology of local flame-flame interactions in turbulent flames, Combust. Flame 203, 92 (2019).

[46] A. W. Skiba, T. M. Wabel, C. D. Carter, S. D. Hammack, J. E. Temme, T. Lee, and J. F. Driscoll, Reaction layer visualization: A comparison of two PLIF techniques and advantages of kHz-imaging, Proc. Combust. Inst. 36, 4593 (2017).

[47] J. Milnor, Analysis of Mathematical Studies (Princeton University Press, Princeton, NJ, 1963).

[48] W. H. Press, S. A. Teukolsky, W. T. Vetterling, and B. P. Flannery, Numerical Recipes, 3rd ed. (Cambridge University Press, Cambridge, UK, 2007).

[49] R. S. Cant, Senga2: User Guide, Technical Report CUED-THERMO-2012/04-2, Cambridge University Engineering Department.

[50] J. Li, Z. Zhao, A. Kazakov, and F. L. Dryer, An updated comprehensive kinetic model of hydrogen combustion, Int. J. Chem. Kinet. 36, 566 (2004).

[51] J. H. Chen, A. Choudhary, B. de Supinski, M. DeVries, E. R. Hawkes, S. Klasky, W. K. Liao, K. L. Ma, J. Mellor-Crummey, N. Podhorszki, R. Sankaran, S. Shende, and C. S. Yoo, Terascale direct numerical simulations of turbulent combustion using S3D, Comput. Sci. Discov. 2, 015001 (2009).

[52] S. Chaudhuri, H. Kolla, H. L. Dave, E. R. Hawkes, J. H. Chen, and C. K. Law, Flame thickness and conditional scalar dissipation rate in a premixed temporal turbulent reacting jet, Combust. Flame 184, 273 (2017).

[53] S. B. Pope, P. K. Yeung, and S. S. Girimaji, The curvature of material surfaces in isotropic turbulence, Phys. Fluids A 1, 2010 (1989).

[54] R. S. Cant, C. Rutland, and A. Trouve, Statistics for laminar flamelet modeling, in Proceedings of the Summer Program (Centre for Turbulence Research, Stanford University/NASA-AMES, 1990), pp. 299-310.

[55] N. Chakraborty, M. Katragadda, and R. S. Cant, Statistics and modelling of turbulent kinetic energy transport in different regimes of premixed combustion, Flow Turbul. Combust. 87, 205 (2011).

[56] T. S. Lundgren, Linearly forced isotropic turbulence, in Annual Research Briefs (CTR Stanford, Stanford, CA, 2003), pp. 461-473.

[57] M. Klein, N. Chakraborty, and S. Ketterl, A comparison of strategies for direct numerical simulation of turbulence chemistry interaction in generic planar turbulent premixed flames, Flow Turbul. Combust. 99, 955 (2017).

[58] A. Tyagi, I. Boxx, S. Peluso, and J. O'Connor, Pocket formation and behavior in turbulent premixed flames, Combust. Flame 211, 312 (2020).

[59] T. M. Wabel, A. W. Skiba, and J. F. Driscoll, Turbulent burning velocity measurements: Extended to extreme levels of turbulence, Proc. Combust. Inst. 36, 1809 (2017). 